\title{
Nonparaxial pulse broadening in a solenoid focusing element
}

\author{
D. P. Grote* and A. Friedman ${ }^{\dagger}$ \\ Lawrence Livermore National Laboratory, 7000 East Avenue, Livermore, California 94550, USA
}

\author{
E. P. Lee
}

Lawrence Berkeley National Lab, 1 Cyclotron Road Mail Stop 47R0112, Berkeley, California 94720-8201, USA

(Received 22 February 2012; published 24 October 2012)

\begin{abstract}
In a standard scenario for focusing an ion beam onto a target, for example with ion beam driven inertial fusion energy, the beam is compressed longitudinally by a velocity ramp to enhance the current and then directed through a transverse focusing system to produce a small, bright spot on the target. To reach the highest levels of compression, the space-charge of the beam is neutralized, typically by the presence of a plasma with a density greater than the beam density. The system is arranged so that the peak longitudinal compression is coincident with the minimum transverse spot size. In this scenario, it has been discovered that nonparaxial effects can lead to degradation in the amount of compression. The transverse focusing causes a radially dependent variation in the axial velocity of the ions, leading to a radially dependent time delay that degrades the peak compression. This effect, nonparaxial pulse broadening, can become significant for short pulses and large focusing fields - the time delay can be comparable to the final pulse length. This pulse broadening will be present in both solenoid and quadrupole focusing systems. This paper describes this effect in solenoids, with some examples. It is expected that the size of the effect will be comparable with quadrupole focusing.
\end{abstract}

DOI: 10.1103/PhysRevSTAB.15.104001

PACS numbers: 29.27.Eg, 52.59.Sa

\section{INTRODUCTION}

Ion beams can be used to heat targets to study warm dense matter [1] and high energy density physics, and can be used to drive targets for fusion energy [2]. A common technique used to enhance the beam power is compressing the beam longitudinally, via a velocity ramp, or "tilt", with the tail of the beam moving faster than the head, before it is focused onto the target. To maximize the compression, the space-charge of the beam can be neutralized. The pipe where the compression takes place is filled with a plasma that has a density higher than the beam density. The neutralization prevents the space-charge blow off from the head and tail of the beam and prevents the space-charge from inhibiting the compression, especially near the peak when the space-charge forces would be the largest. The neutralization also prevents the transverse expansion due to space-charge, removing the need for transverse confinement before the final focus. Note that the presence of the plasma can affect the beam through collisions, for example via scattering and ionization, especially at the driver scale for fusion energy. In present experiments, however, the

\footnotetext{
*dpgrote@lbl.gov

tafriedman@lbl.gov

¥eplee@lbl.gov
}

Published by the American Physical Society under the terms of the Creative Commons Attribution 3.0 License. Further distribution of this work must maintain attribution to the author(s) and the published article's title, journal citation, and DOI. plasma densities are low enough that the collisions are negligible and so are not considered here. To obtain the shortest pulse durations, the compression is arranged to reach its peak at the same point when the beam is focused transversely down into a small spot, when the beam strikes the target.

It is known that having a velocity tilt in the final focus can degrade the focal spot size, the chromatic aberration, because of differing focal lengths for the different velocities. An analogous effect has recently been discovered, the degradation of the compression because of radially dependent velocity, a nonparaxial effect. In a solenoid focusing system, the particles develop radial dependent rotational and radial velocities at the expense of longitudinal velocity, causing a radial dependent time delay. In a quadrupole, the particles similarly develop a transverse dependent velocity, from the alternating gradient flutter and the focusing, which would lead to a transverse dependent time delay. If this delay is comparable to the final pulse duration the compression will be degraded. In the typical focusing used in the bulk of the accelerator, this time delay is present but negligible because of the smaller solenoid strengths, much longer pulse duration; also, the beam is closer to Brillouin flow where the axial velocity is independent of radius. In the final focus, however, the field is much stronger, focusing the beam to a small spot, and the beam duration is much shorter, so the time delay can become comparable to the pulse duration. Also, as the beam is neutralized, the radial variation of the longitudinal velocity due to the space-charge potential 
is to some degree removed (depending on the detailed scenario).

When optimizing the power of the beam on the target, because of the chromatic aberration, there is a trade-off in the amount of velocity tilt. More tilt improves the peak compression, but degrades the focal spot size. With nonparaxial pulse broadening, there is a similar trade-off in the focusing strength of the final focus optic. A stronger focus gives a smaller spot, but degrades the compression. There is a further trade-off. As will be shown below, the degradation of the compression diminishes with a smaller beam radius in the final focus optic, but with a smaller beam radius, the focal length is shorter, moving the target closer to the solenoid and into the increased magnetic fringe fields. These trade-offs need to be taken into account designing the final focus system.

\section{ESTIMATION OF THE DELAY IN SOLENOIDS}

Estimates of the amount of delay can be made. In a solenoid focusing system, the amount of time delay accumulates both inside of the solenoid and after the beam exits. Inside of the solenoid, the delay occurs because of the combination of rotational velocity that the particles pick up from the force $F_{\theta} \propto v_{z} \times B_{r}$ and the resulting radial velocity from the force $F_{r} \propto v_{\theta} \times B_{z}$. After exiting, the resulting radial velocity of the focus further increases the delay. With the conservation of kinetic energy of a particle in a magnetic field, the $v_{\theta}$ and $v_{r}$ that develop lead to a decrease in $v_{z}$. The estimates below ignore spacecharge effects, assuming that the beam is fully neutralized.

\section{A. Collimated beam}

As a beam enters a solenoid, it spins up because of the $B_{r}$, obtaining an angular velocity of $\omega_{c} / 2$, where $\omega_{c}$ is the cyclotron frequency $\omega_{c}=e B_{z} / m$, with $B_{z}$ being the magnetic field on axis and $e$ and $m$ being the particle charge and mass. Because of this $v_{\theta}$, the particles will begin to move radially as well. Simple conservation of energy gives the longitudinal velocity,

$$
v_{z}^{2}=v_{0}^{2}-v_{\theta}^{2}-v_{r}^{2}=v_{0}^{2}-\frac{r^{2} \omega_{c}^{2}}{4}-v_{r}^{2}
$$

where $v_{0}$ is the longitudinal velocity of the particle before entering the solenoid. For simplicity, the particles are assumed to have no transverse velocity initially. Assuming a hard edged field model for the solenoid, one can obtain the equation of motion of the particles (see for example [3], Eq. 3.145):

$$
\begin{gathered}
r=r_{0} \cos k z, \\
r^{\prime}=-r_{0} k \sin k z .
\end{gathered}
$$

Here, $r^{\prime}=v_{r} / v_{z}, r_{0}$ is the initial radius of the particle, and $k=\omega_{c} / 2 v_{0}$. Inserting this into Eq. (1) gives the following:

$$
\begin{aligned}
v_{z}^{2} & =v_{0}^{2}-\frac{r_{0}^{2} \omega_{c}^{2}}{4} \cos ^{2} k z-\frac{r_{0}^{2} \omega_{c}^{2}}{4} \sin ^{2} k z \\
& =v_{0}^{2}-\frac{r_{0}^{2} \omega_{c}^{2}}{4} .
\end{aligned}
$$

Note that this gives a $v_{z}$ that is independent of $z$. Assuming that the transverse velocity is small compared to $v_{0}, v_{z}$ can be approximated:

$$
v_{z} \approx v_{0}\left(1.0-\frac{r_{0}^{2} \omega_{c}^{2}}{8 v_{0}^{2}}\right) .
$$

For this first component, the time delay of particles off axis compared to a particle on axis, $\Delta t_{1}$, can be estimated given the length of the solenoid, $L_{s}$. The maximum value of the time delay occurs for particles at the outer edge of the beam, having $r_{0}=a$, where $a$ is the beam radius. The time delay is the difference in the time it takes an on axis particle (which has no delay) and an edge particle to traverse the length of the solenoid, $\Delta t_{1}=L_{s} / v_{0}$ $L_{s} / v_{z}$. With $v_{\theta} \ll v_{0}$, using the above, and with further approximation, $\Delta t_{1}$ can be written

$$
\begin{gathered}
\Delta t_{1} \approx \frac{L_{s}}{v_{0}}-\frac{L_{s}}{v_{0}\left(1.0-r_{0}^{2} \omega_{c}^{2} / 8 v_{0}^{2}\right)}, \\
\Delta t_{1} \approx \frac{L_{s} a^{2} \omega_{c}^{2}}{8 v_{0}^{3}} .
\end{gathered}
$$

From Eq. (3) above, $a^{\prime}$, the convergence angle of the beam at the end of the solenoid, can be written

$$
a^{\prime}=-a k \sin k L_{s} .
$$

Incorporating this into the expression for $\Delta t_{1}$ gives a more useful expression,

$$
\Delta t_{1} \approx-\frac{a a^{\prime}}{2 v_{0}} \frac{k L_{s}}{\sin k L_{s}} .
$$

The product $k L_{s}$ gives the total rotation angle in the solenoid. One sees readily that changing the solenoid parameters alone, without changing the beam envelope, cannot significantly affect this component of the time delay.

The second component of the time delay occurs after the focusing beam exits the solenoid, when the beam is converging. The sign of $B_{r}$ is opposite to that in the entrance, causing the particles to spin down, losing their angular velocity. Using a similar derivation as above, starting with the radial velocity $v_{r}=r^{\prime} v_{0}$, the longitudinal velocity is given as follows from the conservation of energy:

$$
v_{z}^{2}=v_{0}^{2}-r^{\prime 2} v_{0}^{2}
$$

This can be approximated for $v_{r} \ll v_{0}$ :

$$
v_{z} \approx v_{0}\left(1-\frac{1}{2} r^{\prime 2}\right)
$$


Again, the maximum time delay will be at the edge of the beam, with $r^{\prime}=a^{\prime}$. After the beam has traveled a distance $z$ from the solenoid, the time delay for off axis particles will be $\Delta t_{2}=z / v_{0}-z / v_{z}$. An approximate value can be given for small $v_{r}$ :

$$
\Delta t_{2} \approx \frac{z a^{\prime 2}}{2 v_{0}} \text {. }
$$

The most interesting case is where the beam reaches peak focusing, at $z=-a \cos \left(k L_{s}\right) / a^{\prime}$. The time delay can be written in a form similar to the form given above for $\Delta t_{1}$ :

$$
\Delta t_{2} \approx-\frac{a a^{\prime}}{2 v_{0}} \cos k L_{s} .
$$

The total maximum time delay is the sum of the two components:

$\Delta t_{\max }=\Delta t_{1}+\Delta t_{2} \approx-\frac{a a^{\prime}}{2 v_{0}}\left(\frac{k L_{s}}{\sin k L_{s}}+\cos k L_{s}\right)$.

This can be expanded to second order in $k L_{s}$ :

$$
\Delta t_{\max } \approx-\frac{a a^{\prime}}{v_{0}}\left(1-\frac{1}{6}\left(k L_{s}\right)^{2}\right) .
$$

Trade-offs are needed in order to reduce the time delay. One would be to reduce the convergence angle, at the cost of an increased spot size. Another is decreasing the beam size, which leads to a shorter focal length. This may go against constraints on the amount of clearance needed between the target plane and the solenoid and will put the target further into the solenoid field which may interfere with the experiment and/or make it more difficult to buck out the fields if that is needed.

The above derivations have been for the maximum time delay. Of more interest is an average of the time delay. This can be calculated in a straightforward manner assuming a cold beam. For a single slice of beam that is monoenergetic and has initially no transverse motion, the distribution at the focus can be written $f=\delta\left(t-\Delta t\left(r_{0}\right)\right) /\left(a^{2} / 2\right)$ for $r_{0} \leq a$, which normalizes to one when integrated over all time and all transverse space. Note that $t=0$ is the time when the on-axis particles reach the focus. The time delay can be written in terms of only $r_{0}$, in addition to the fixed quantities $\omega_{c}, L_{s}$, and $v_{0}$ :

$$
\Delta t \approx r_{0}^{2} \frac{k}{2 v_{0}}\left(k L_{s}+\sin k L_{s} \cos k L_{s}\right) .
$$

With the distribution $f$ defined, the average time and time squared can be calculated:

$$
\begin{aligned}
\bar{t} & =\frac{2}{a^{2}} \int_{0}^{a} \int_{-\infty}^{+\infty} t \delta(t-\Delta t) r_{0} \partial r_{0} \partial t \\
& \approx \frac{1}{2} \Delta t_{\max }
\end{aligned}
$$

$$
\begin{aligned}
\vec{t}^{2} & =\frac{2}{a^{2}} \int_{0}^{a} \int_{-\infty}^{+\infty} t^{2} \delta(t-\Delta t) r_{0} \partial r_{0} \partial t \\
& \approx \frac{1}{3} \Delta t_{\max }^{2} .
\end{aligned}
$$

The rms can then be written

$$
\Delta t_{\mathrm{rms}}=\langle t\rangle=\sqrt{\bar{t}^{2}-\bar{t}^{2}} \approx \sqrt{\frac{1}{12}} \Delta t_{\max }
$$

\section{B. With transverse divergence}

The effect of an nonzero beam divergence when entering the solenoid can be included in the estimate of the nonparaxial pulse broadening. The envelope equations (2) and (3) can be extended to include an initial divergence:

$$
\begin{gathered}
r=r_{0} \cos k z+\frac{r_{0}^{\prime}}{k} \sin k z, \\
r^{\prime}=-r_{0} k \sin k z+r_{0}^{\prime} \cos k z .
\end{gathered}
$$

$r_{0}$ is the radius and $r_{0}^{\prime}$ is the divergence when the beam enters the solenoid. Following the same procedure as above, including the initial divergence in the initial beam energy, the two time delays can be derived. During transport in the solenoid, Eqs. (23) and (24), along with $v_{\theta}=r v_{0} k$, are used with conservation of energy to give the following expression:

$$
\begin{aligned}
& \boldsymbol{v}_{0}^{2}+r^{\prime 2} v_{0}^{2}=v_{z}^{2}+v_{r}^{2}+v_{\theta}^{2}, \\
v_{z}^{2}= & v_{0}^{2}\left(1+r_{0}^{\prime 2}-r_{0}^{2} k^{2} \sin ^{2} k z-r_{0}^{\prime 2} \cos ^{2} k z\right. \\
+ & r_{0} r_{0}^{\prime} \cos k z \sin k z-r_{0}^{2} k^{2} \cos ^{2} k z \\
- & \left.r_{0}^{\prime 2} \sin ^{2} k z-r_{0} r_{0}^{\prime} \cos k z \sin k z\right) .
\end{aligned}
$$

With good cancellations, this reduces to the same result as in Eq. (5), and thus the delay is the same. The energy associated with the initial radial velocity becomes associated with the angular velocity and thus does not affect the longitudinal velocity and the time delay. The beam radius and divergence at the end of the solenoid can be written as follows:

$$
\begin{gathered}
r_{e}=r_{0} \cos k L_{s}+\frac{r_{0}^{\prime}}{k} \sin k L_{s}, \\
r_{e}^{\prime}=-r_{0} k \sin k L_{s}+r_{0}^{\prime} \cos k L_{s} .
\end{gathered}
$$

Following the same procedure as in Eqs. (11) and (12), the time delay at peak focus can be derived. The time delays can be written out,

$$
\Delta t_{1} \approx \frac{L_{s}}{2 v_{0}} r_{0}^{2} k^{2}
$$




$$
\Delta t_{2} \approx \frac{1}{2 v_{0}} r_{e} r_{e}^{\prime}\left(1-\frac{r_{0}^{\prime 2}}{r_{e}^{\prime 2}}\right)
$$

The time delay after the beam leaves the solenoid does not have a simple form in terms of the initial parameters. The radial velocity at the exit of the solenoid depends on the initial radial velocity, and so it affects the time delay. Note that it is expected that typically $r_{0}^{\prime 2} \ll r_{e}^{\prime 2}$, so the second term, the ratio $r_{0}^{\prime 2} / r_{e}^{\prime 2}$, will generally be small for a final focus solenoid. Similarly, the effect of $r_{0}^{\prime}$ on the term $r_{e} r_{e}^{\prime}$ will typically be small for the same reason.

\section{SIMULATION}

The analytic results from above can be compared against simulation. The simplest test is simulation of a beam that has the distribution described above, a monoenergetic slice of beam, but with a realistic solenoid profile (not hard edged). The beam is completely neutralized. The beam is initialized before the solenoid, and followed through to the point of focus. The example beam is singly charged lithium ions with an energy $1.65 \mathrm{MeV}$. The initial radius is $2 \mathrm{~cm}$. The simulations were done with the code WARP [4], a particle-in-cell code designed to be used for high current, space-charge limited accelerators.

Simulations were done with two different profiles for the solenoid, as shown in Fig. 1. The black curve is from the expression for the field on axis for a cylindrical current sheet with surface current density $K_{\theta}$, radius $R$, and length $l$ :

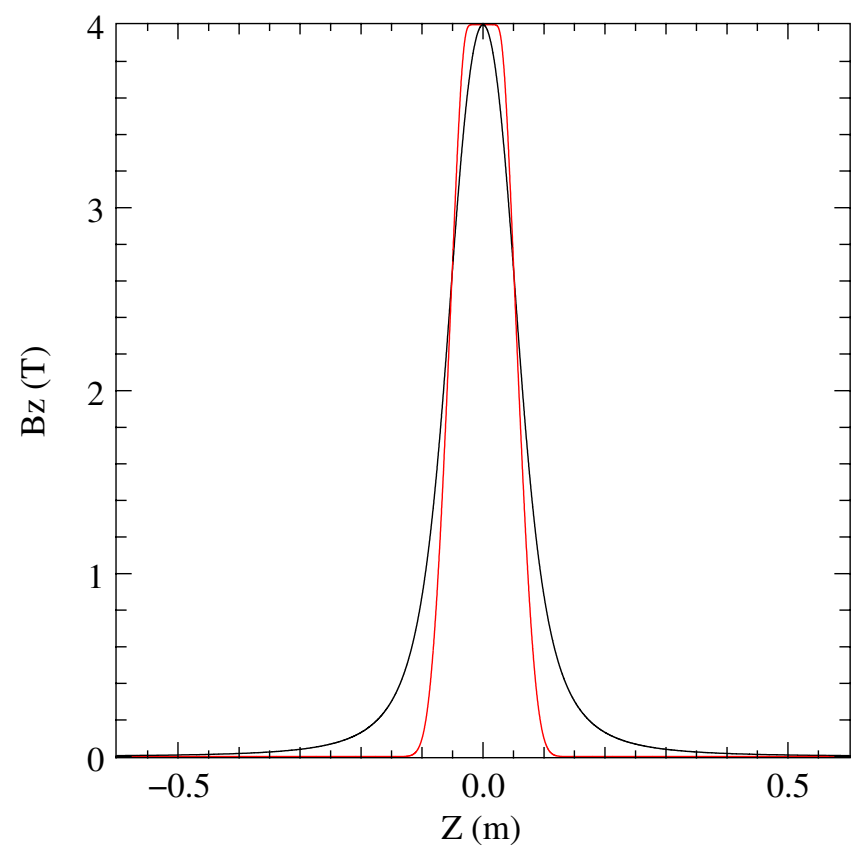

FIG. 1. The two longitudinal profiles of the solenoid field used in the simulations. The black is the profile that is mostly fringed, and the red is closer to a hard edge.
$B_{z}(z)=\frac{K_{\theta} \mu_{0}}{2}\left[\frac{l-2 z}{\sqrt{4 R^{2}+(l-2 z)^{2}}}+\frac{l+2 z}{\sqrt{4 R^{2}+(l+2 z)^{2}}}\right]$.

For the simulations, the radius $R=5.9 \mathrm{~cm}$ and length $l=10 \mathrm{~cm}$. The surface current density was chosen to give the specified field on axis at the center of the solenoid. The length of the solenoid is comparable to the diameter, so the field is essentially all fringe. The red curve was artificially generated to be closer to a hard edged profileit might be what is produced in the presence of shielding, for example. The profile was adjusted so that both cases have the same value of the integral of $B_{z}^{2}$-both have the same effective length of $10 \mathrm{~cm}$. In both cases, higher order components of the field with up to the third derivative of $B_{z}$ on axis were included in the simulations.

\section{A. Without longitudinal velocity tilt}

The results of the simulations without longitudinal velocity tilt are shown in Fig. 2. The circle and plus sign markers show the results from the simulations, the rms time of the particles at the focal spot, for varying solenoid field. The focal spot is defined as the location where the radial rms size of the beam is smallest. This location does not coincide exactly with the focal spot location calculated analytically with the hard edge field profile. The circles are for the case that is nearly all fringe and the plus signs are

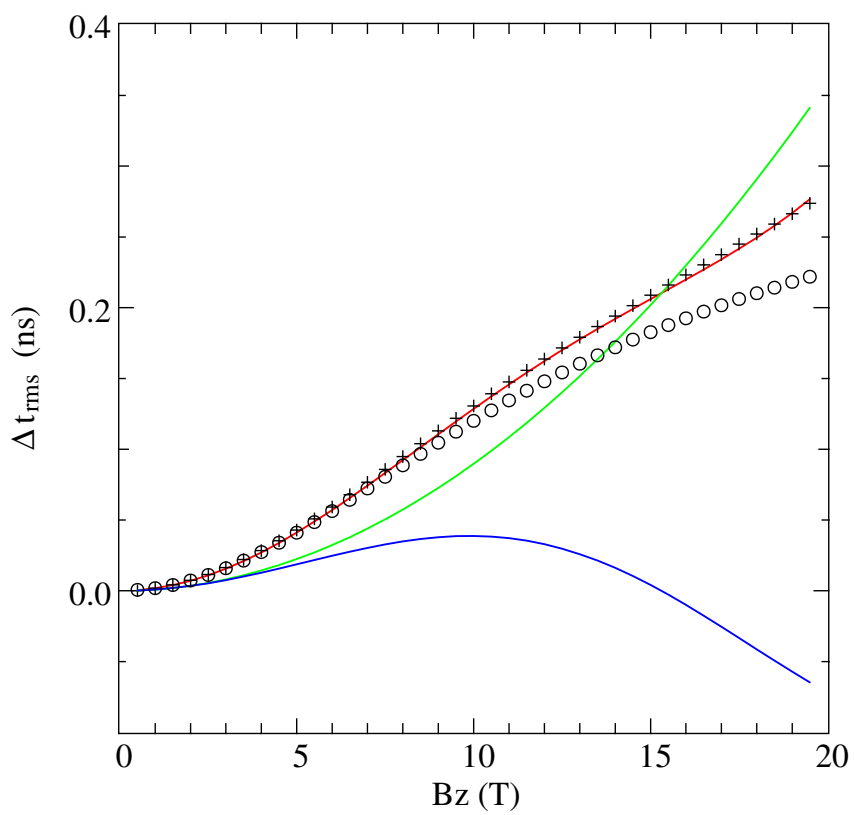

FIG. 2. Comparison of $\Delta t_{\text {rms }}$ from simulation with the analytic result. The circles show the rms time at the focal spot in the simulation for the case where the solenoid is mostly fringe. The plus signs are for the case where the solenoid is nearly hard edged. The red curve is $\Delta t_{\mathrm{rms}}$. The green curve is $\Delta t_{1 \mathrm{rms}}$ and the blue is $\Delta t_{2 \mathrm{rms}}$. Note that beyond $B_{z}=15 \mathrm{~T}$, the focal spot is inside of the solenoid, hence the negative values for $\Delta t_{2 \mathrm{rms}}$. 
for the case that is nearly hard edged. The red curve shows the analytic result for $\Delta t_{\text {rms }}$, from Eq. (22). The green curve shows $\sqrt{1 / 12} \Delta t_{1}$ and the blue curve $\sqrt{1 / 12} \Delta t_{2}$. Note that at roughly $15 \mathrm{~T}$, the blue curve goes negative-that is the point where the focal spot moves inside of the effective length of the solenoid.

As expected, good agreement with the analytic result was found for the nearly hard edged case. For the nearly all fringe case however, for larger $B_{z}$, the resulting time delay is less than the analytic value. For those cases, the maximum transverse velocities come out to be less than that for the hard edged case. The focal spot is near the same location however, since the beam starts focusing earlier. Also, because of the large extent of the fringe fields, above about $5 \mathrm{~T}$ the focal spot occurs in a location where the fringe field is still significant and the full inward radial velocity had not developed before the beam reaches the focus. Figure 3 shows the evolution of the time delay as a function of beam position, comparing the results from the mostly fringe solenoid profile and the analytic expression. Even though the time delay starts developing earlier than in the hard edged case, the transverse velocities overall remain smaller than in the hard edged case and so the accumulated time delay is less.

A fully realized system would likely have a bucking coil to remove the magnetic field from the area of the target. A nonzero field at the target could affect the behavior of the target and confound the results. Also, in the nonzero $B$

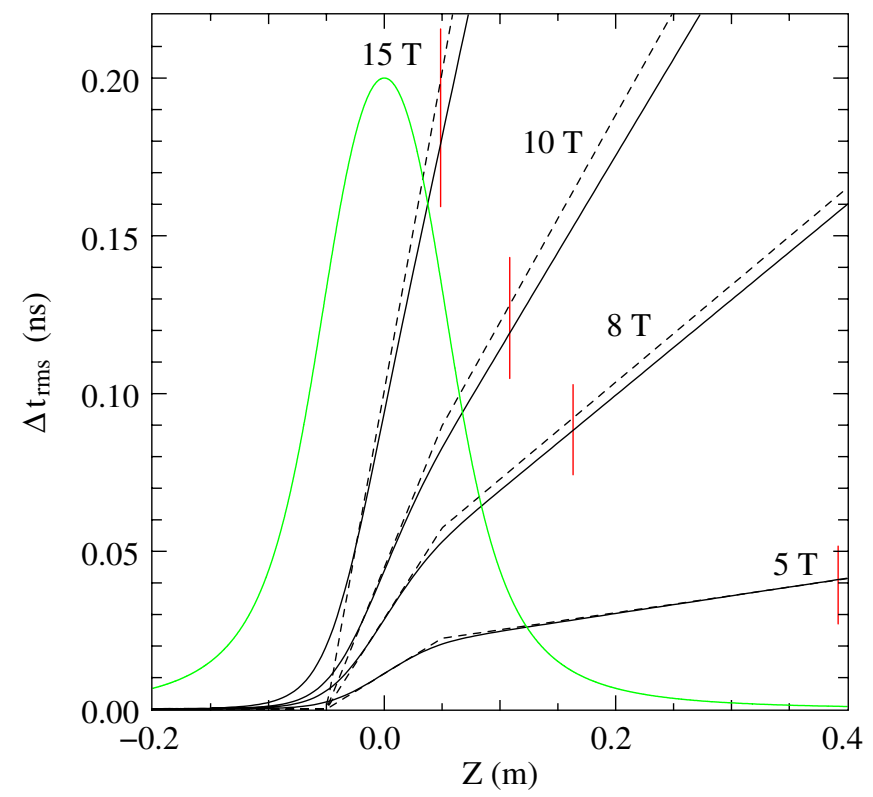

FIG. 3. Comparison of the simulated and analytic calculation of $\Delta t_{\mathrm{rms}}$ for several different solenoid strengths. For each case, the solid line is from the simulation and the dashed line is from the analysis. The red vertical lines show the locations of the focal spots for the simulated cases. The green curve shows the longitudinal profile of the solenoid field, scaled vertically to fit the graph. field, the beam will have a nonzero canonical angular momentum which can limit the focusability of the beam, leaving a larger spot size than would otherwise be achieved if the target were field free. With a bucking coil, the time delay would likely end up between the hard edged case and the mostly fringe case.

\section{B. With longitudinal velocity tilt}

A next test case is a full length beam with velocity tilt. Cases are simulated with zero thermal velocity spread and with realistic thermal velocity spreads. The same singly charged, $1.65 \mathrm{MeV}$ lithium beam is used. The beam has an initial length $l_{\text {init }}=10 \mathrm{~cm}$ and a velocity tilt $\Delta v / v=$ $\left(v_{\text {tail }}-v_{\text {head }}\right) / v_{\text {center }}=0.033$. Without the final focus solenoid, the beam would reach a maximum longitudinal compression in $l_{\text {init }} / \Delta v / v=3.03$ meters. With the solenoid in place, however, because of the effects of the nonparaxial pulse broadening, the location of the peak compression will vary, always happening earlier. In the simulations, for each value of the solenoid focusing strength, an iteration is done adjusting the location of the solenoid until the location where the peak current occurs coincides with the location of the minimum rms spot radius. For the cases here, the location of peak compression can vary by as much as $10 \mathrm{~cm}$ or more. The solenoid that is mostly fringe field was used.

The results with no thermal spread are shown in Fig. 4. With no thermal spread and no solenoid, the pulse duration at peak compression would be zero. For small values of the

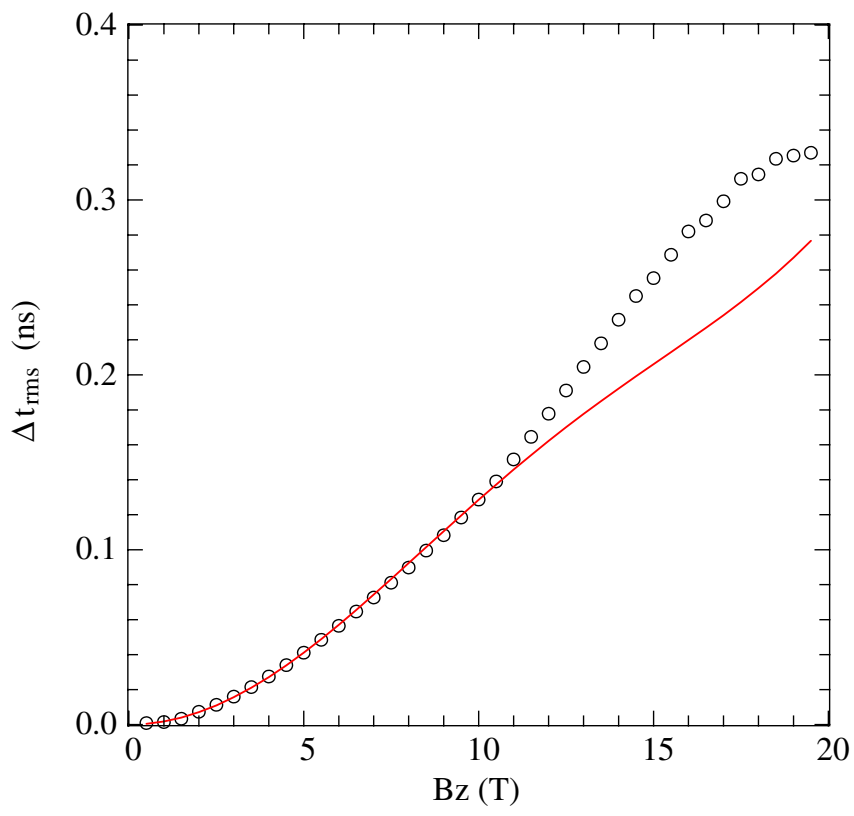

FIG. 4. Comparison of the simulated (circles) and analytic calculation (red curve) of $\Delta t_{\mathrm{rms}}$ for a beam with finite extent and an axial velocity tilt. The calculation of $\Delta t_{\text {rms }}$ does not take into account the tilt. The solenoid is the case that is mostly fringe. 
solenoid focusing, it is seen that the effect on the pulse duration is the same as if there was no tilt. For larger values, the point of peak compression moves upstream, leaving some residual tilt in the beam. This residual increases the minimum pulse duration above what is there with only the time delay.

Results with thermal spread are shown in Fig. 5. The normalized edge emittance of the beam is taken to be $2 \pi \mathrm{mm} \mathrm{mrad}$, and the longitudinal velocity spread equal to 0.3 times the transverse velocity spread. The black circles show the results from the simulations with the mostly fringe solenoid and the plus signs from the nearly hard edged solenoid. Note that there is some variation in the results due to statistical noise in the particles because of the randomness in the initial particle velocity due to the thermal velocity spread. The difference in the results for the two different solenoid profiles is consistent with the results found with no tilt or thermal spread. The red curve shows the analytic results without tilt and thermal spread. The green curve shows the same analytic data shifted upwards to have the same $\Delta t_{\mathrm{rms}}$ at zero focusing field. The blue curve is the geometric mean of the $\Delta t_{\text {rms }}$ at zero focusing field and the analytic result. For all focusing strengths, the final pulse duration is bounded by the sum and the geometric sum. For modest focusing field, the focal spot is beyond the end of the solenoid. As shown in Fig. 6,

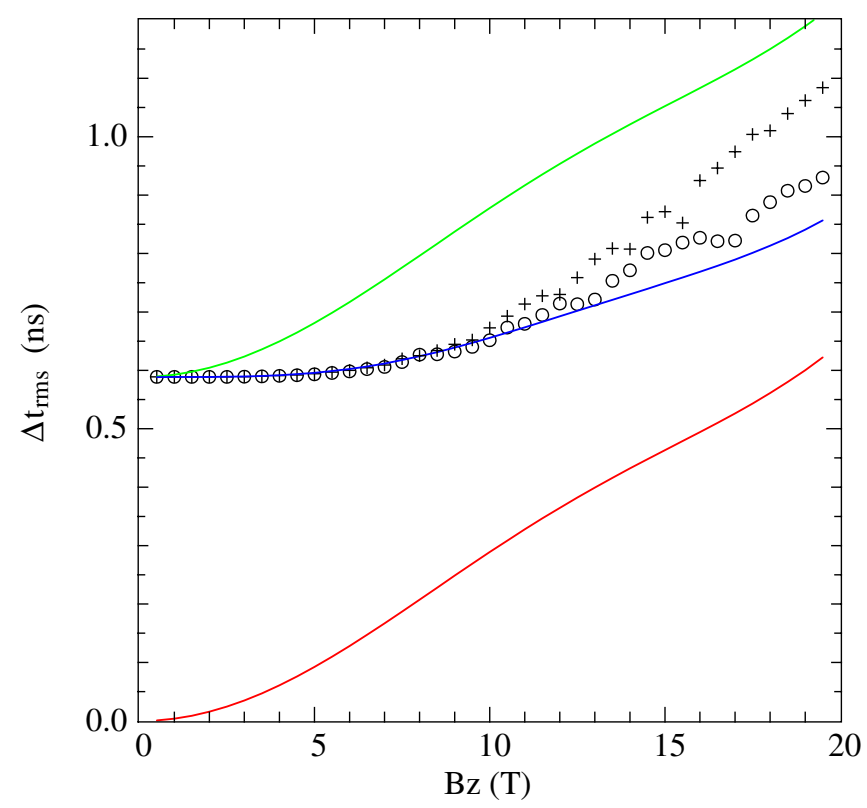

FIG. 5. Comparison of the simulated (circles and pluses) and analytic calculation (red, green, and blue curves) of $\Delta t_{\mathrm{rms}}$ for a beam with finite extent, axial velocity tilt, and normalized edge emittance of $2 \pi \mathrm{mm}$ mrad. The circles are from the case with the solenoid that is mostly fringe, and the plusses from the solenoid that is nearly hard edged. The green curve is the sum and the blue the geometric sum of the analytic solution and $\Delta t_{\mathrm{rms}}$ from the simulation with no solenoid field. The analytic $\Delta t_{\mathrm{rms}}$ does not include either the tilt or the thermal spread.

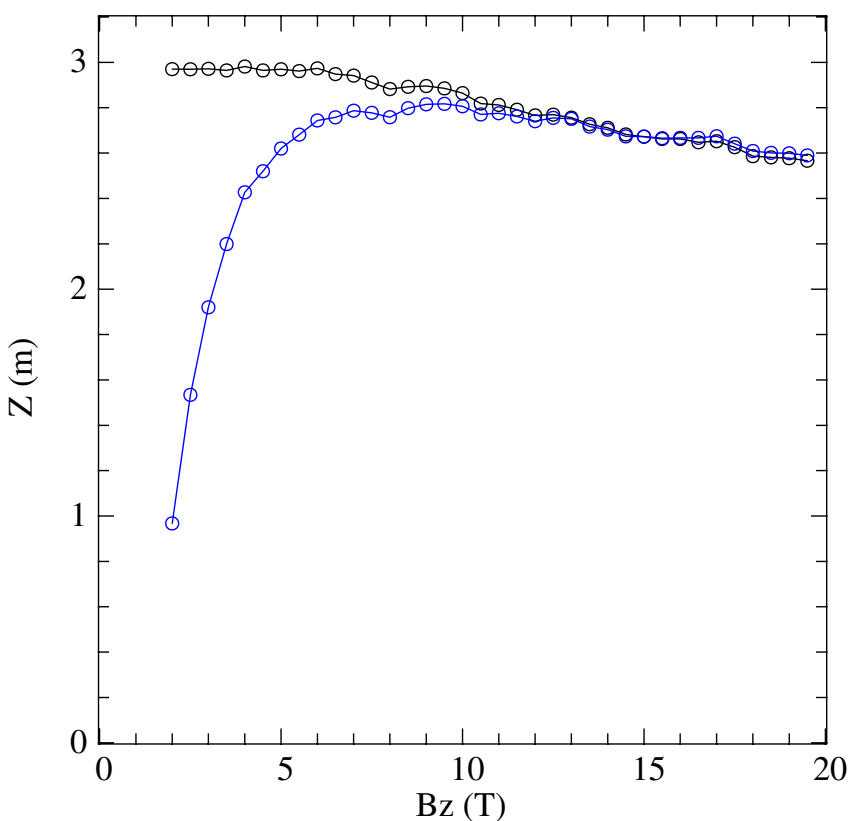

FIG. 6. The location of the solenoid end (blue) and the focal spot and peak compression (black) from the simulation with velocity tilt and thermal spread.

in this case modest is up to around $B_{z} \approx 10 \mathrm{~T}$. Figure 7 shows the results from the cases with the normalized edge emittances of 4 and $8 \pi \mathrm{mm}$ mrad, which exhibit similar behavior. For these particular cases, the spread in the pulse

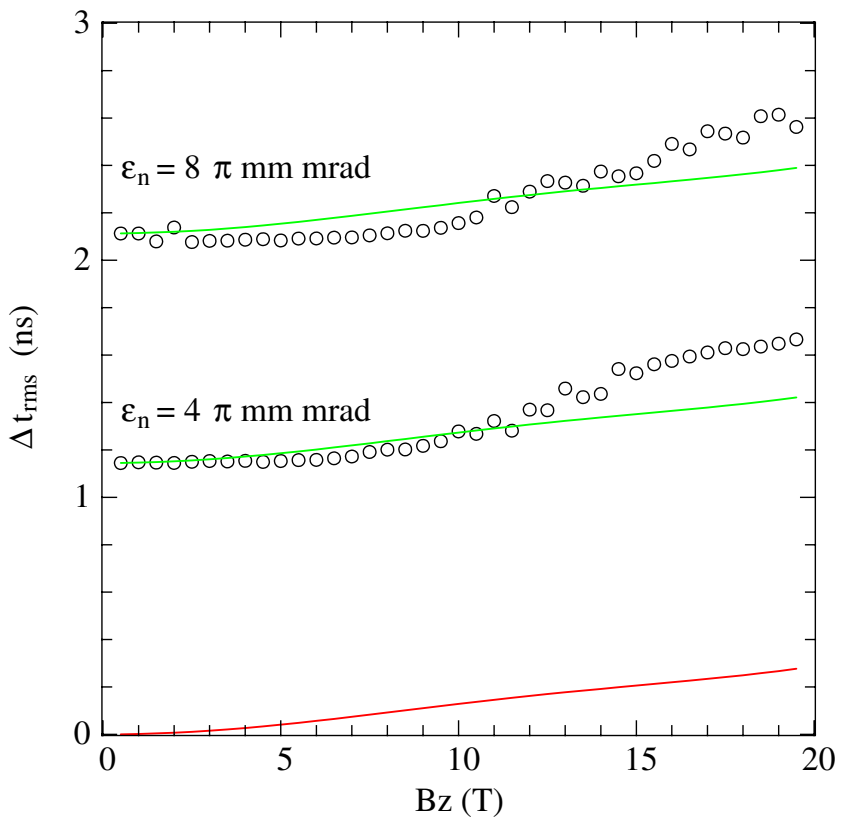

FIG. 7. Comparison of the simulated (circles) and analytic calculation (green curve) of $\Delta t_{\mathrm{rms}}$ for beams with finite extent, axial velocity tilt, and normalized edge emittances of 4 and $8 \pi \mathrm{mm}$ mrad. In each case, the green curve is the analytic $\Delta t_{\mathrm{rms}}$ with the $\Delta t_{\mathrm{rms}}$ from the simulation with small solenoid field added. The analytic $\Delta t_{\text {rms }}$ does not include either the tilt or the thermal spread. The solenoid is the case that is mostly fringe. 


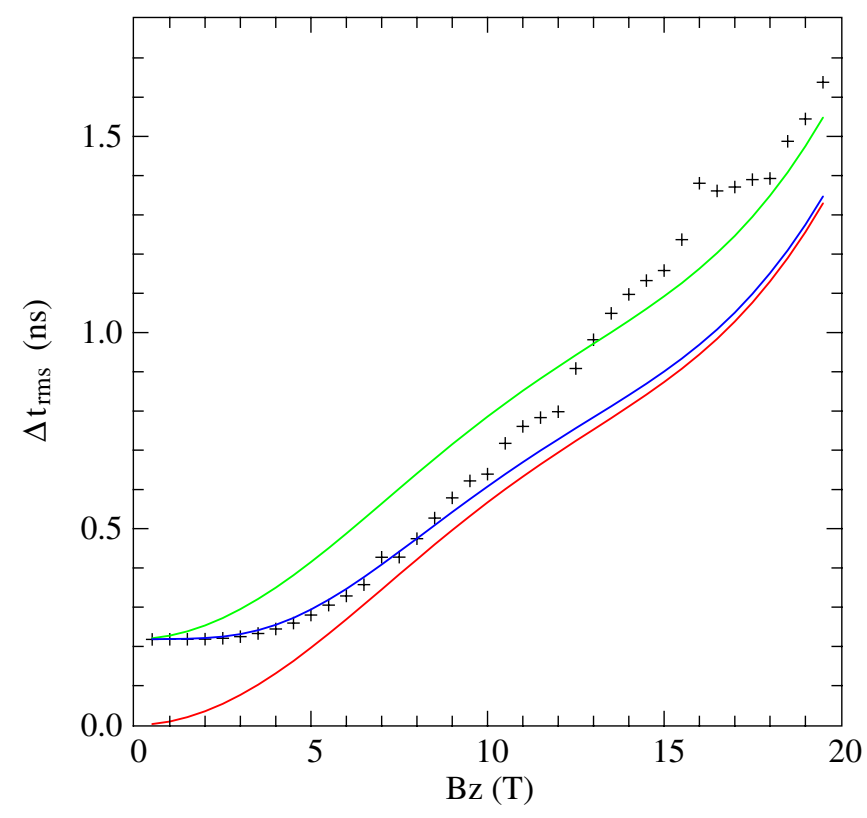

FIG. 8. Comparison of the simulated (plusses) and analytic calculation (red, green, and blue curves) of $\Delta t_{\mathrm{rms}}$ for a beam with finite extent, axial velocity tilt, normalized edge emittance of $2 \pi \mathrm{mm}$ mrad, $3.5 \mathrm{~cm}$ radius, and $1.2 \mathrm{MeV}$. The solenoid is nearly hard edged. The green curve is the sum and the blue the geometric sum of the analytic solution and $\Delta t_{\mathrm{rms}}$ from the simulation with no solenoid field. The analytic $\Delta t_{\mathrm{rms}}$ does not include either the tilt or the thermal spread.

due to the nonparaxial effect comes out small compared to the effect of the thermal spread. Figure 8 shows another case typical of current experiment parameters, with lower beam energy and larger initial radius.

A full design of the focusing system would optimize some measure of the beam intensity on target, which would depend on both the focal spot size and the pulse duration. There will be an optimal focusing strength giving the best intensity. A smaller focusing strength would give a shorter pulse duration but larger spot size. A larger focusing strength would give a smaller spot size but a larger pulse duration. If there is a bucking coil, it would have to be included in the optimization since it would affect the value of the optimum.

\section{CONCLUSION}

A new nonparaxial effect has been described. This effect causes broadening of beam pulses during the process of focusing the pulse onto a small spot, and becomes important at short pulse durations. The broadening is due to a time delay that occurs because of the radially dependent transfer of kinetic energy from longitudinal to transverse directions from the action of the focusing element and the resulting radial convergence. This puts a constraint on the minimum achievable pulse duration. Analytical estimates of the size of the minimum duration were derived in the limiting case of no velocity tilt and no thermal velocity spread. This was compared to simulation with realistic solenoid profiles and good agreement was found. Further simulations examined the pulse broadening coupled with longitudinal velocity tilt and thermal spread. With velocity tilt, at low to moderate focusing strengths, agreement was found with the analytic result, showing little coupling with the tilt. With thermal spreads, it is seen that adding the analytic result to the pulse duration without focus gives an upper bound to the pulse duration for modest focusing strengths. It is expected that the degradation of the pulse duration will be similar in a quadrupole focusing system.

[1] J. J. Barnard, J. Armijo, R. M. More, A. Friedman, I. Kaganovich, B. G. Logan, M. M. Marinak, G. E. Penn, A. B. Sefkow, P. Santhanam, P. Stoltz, S. Veitzer, and J. S. Wurtele, in Proceedings of the 16th International Symposium on Heavy Ion Inertial Fusion, HIF06 [Nucl. Instrum. Methods Phys. Res., Sect. A 577, 275 (2007)].

[2] W. M. Sharp et al., in Proceedings of the 2011 Particle Accelerator Conference (IEEE, New York, 2011), pp. 1386-1393.

[3] Martin Reiser, Theory and Design of Charged Particle Beams, Wiley Series in Beam Physics and Accelerator Technology (Wiley, New York, 1994).

[4] David P. Grote, AIP Conf. Proc. 749, 55 (2005). 